\title{
The Origins of Digital Image Processing \& Application areas in Digital Image Processing Medical Images
}

\author{
Bhausaheb Shivajirao Shinde, A.R. Dani \\ Computer Science Department, R.B.N.B. College, Shrirampur Affiliated by Pune University \\ Dist. Ahmednagar pin:413709 \\ Maharashtra, India \\ Head, I Square IT Pune \\ Maharashtra, India
}

\begin{abstract}
One of the first application of digital image was in the newspaper industry, when picture were first sent by submarine cable between London and New York.Introducation of the Bartlane cable picture transmission system in the early 1920s reduced the time required to transport a picture across the Atlantic from more than a week to less than three hours.Spaecialized printing equipment coded pictures for cable transmission and then reconstructed them at the receiving end. Some of the initial problems in improving the visual quality of these early digital pictures were related to the selection of printing producers and the distribution of intensity levels. In facts ,digital images requires so much storages and computation power that progress in the field of digital image processing has been dependent on the development of digital computers and of supporting technologies that include data storage, display and transmission. The digital image is composed of a finite number of elements, each of which has a location and values. These elements are referred as picture element, image element \&pixels. Pixels used to denote the element of a digital image. The process of acquiring an image of the area containing the text, preprocessing that image, extracting the individual characters, describing the character in the form suitable for computer processing \& recognizing those individual character are Digital Image Processing.
\end{abstract}

Keyword: MRI - Magnetic Resonance Imaging ,IPT EM Electron Microscopy, CT - Computerized Tomography, CAT - Computerized Axial Tomography, PC - Personal Computer

\section{INTRODUCTION:}

\section{What is Digital Image Processing?}

Digital image processing allows one to enhance image features of interest while attenuating details irreverent to a given application, and then extract useful information about the scene from the enhanced image. An image may be defined as a two-dimensional function, $\mathrm{f}(\mathrm{x}, \mathrm{y})$, where $\mathrm{x}$ and $\mathrm{y}$ are spatial (plane) co-ordinate, and amplitude of $\mathrm{f}$ at any pair of co-ordinates $(\mathrm{x}, \mathrm{y})$ is called the intensity or gray level of the image at that point. When $\mathrm{x}, \mathrm{y}$ and the amplitude values of $\mathrm{f}$ are all finite, discrete quantities, we call the image a digital image. The field of digital image processing refers to processing digital images by means of a digital computer. A digital image is composed of a finite number of elements, each of which has a particular location and values. These elements are referred to as picture elements, image elements and pixels. Pixels are the term most widely used to denote the element of digital image

\section{The Origins of Digital Image Processing:}

One of the first application of digital image was in the newspaper industry, when picture were first sent by submarine cable between London and New York.Introducation of the Bart lane cable picture transmission system in the early 1920 s reduced the time required to transport a picture across the Atlantic from more than a week to less than three hours.Spaecialized printing equipment coded pictures for cable transmission and then reconstructed them at the receiving end. Some of the initial problems in improving the visual quality of these early digital pictures were related to the selection of printing producers and the distribution of intensity levels. In facts ,digital images requires so much storages and computation power that progress in the field of digital image processing has been dependent on the development of digital computers and of supporting technologies that include data storage, display and transmission. The digital image is composed of a finite number of elements, each of which has a location and values. These elements are referred as picture element, image element \&pixels. Pixels used to denote the element of a digital image. The process of acquiring an image of the area containing the text, preprocessing that image, extracting the individual characters, describing the character in the form suitable for computer processing \& recognizing those individual character are Digital Image Processing. Digital image processing techniques began in the late 1960s and early 1970 s to be used in medical imaging, remote Earth resource observations and astronomy. The invention in the early 1970s of computerized axial tomography (CAT) also called computerized tomography (CT) is one of the most 


\section{Bhausaheb Shivajirao Shinde, A.R. Dani / IOSR Journal of Engineering (IOSRJEN) \\ www.iosrjen.org}

Vol. 1, Issue 1, pp.066-071

important events of image processing in medical diagnosis. Computerized axial tomography is a process in which a ring of detectors encircles a patient and an X-Ray source, concentric with detector ring, rotates about the patient. The XRay passes through the object and is collected at the opposite end by the corresponding detectors in the ring. As the source rotates, this procedure is repeated. Tomography consist algorithms that use the sensed data to construct an image that represent the 'slice' through the object. Computer procedure are used to enhance the contrast or code the intensity levels into color for easier interpretation of X-Rays and other images used in industry, medicine and the biological sciences. Image enhancement and restoration procedure are used to process degraded images of unrecoverable objects or experimental result too expansive to duplicate. Image processing methods have successfully restored blurred pictures that were the only available records of rare artifacts lost or damaged after being photographed.

\section{Application areas in Digital Image Processing}

Today, there is almost no area of technical endeavor which is not impacted by digital image processing. Many application oriented image analyzers are available and are working satisfactorily in real environment. One of the simplest way to develop a basic understanding of image processing application to categorize images according to there resources e.g. Visual, $\mathrm{X}$-ray and so on. The principal energy source for images in use today is the electromagnetic energy spectrum. Other important sources of energy include ultrasonic and electronic in the form of electron beams used in electron microscopy. In this section we discuss how images are generated in these various categories and the area in which they applied. Images based on radiation from the EM spectrum are most familiar, especially images in the X-ray and visual bands of the spectrum.

\section{X-ray Imaging}

$\mathrm{X}$-ray imaging is perhaps the most familiar type of imaging. $\mathrm{X}$-ray is among the oldest sources of EM radiation used for imaging. The use of X-rays is medical diagnostics, but they also are used extensively in industry and other areas like astronomy. X-ray for medical imaging are generated using Xray tube, which is a vacuum tube with a cathode and anode. The cathode is heated, causing free electrons to be released. These electrons flow at high speed to the positively charged anode. When the electrons strike a nucleus, energy is released in the form of X-ray radiation. Figure (a) shows a familiar chest -ray generated simply by placing the patient between an $\mathrm{X}$-ray source and a film sensitive to X-ray energy.

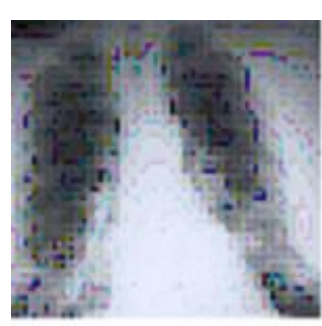

In digital radiography, digital images are obtained by one of two methods: (1) by digitizing X-ray film; or (2) by having the $\mathrm{X}$ ray that passes through the patients fall directly onto devices that Fig (a) X-ray

Convert X-ray to light. The light signal in turn is captured by a light sensitive digitizing system.

Angiography is another major application in an area called contrast enhancement radiography. This is used to obtain image of blood vessels. A catheter is an inserted into a vein in the groin. The catheter is threaded into blood vessel and guided to area to be studied. When the catheter reaches the site under investigation, an X-ray contrast medium is injected through the catheter. This enhances contrast of the blood vessels and enables the radiologist to see any blockages.

\section{Steps in Digital Image Processing}

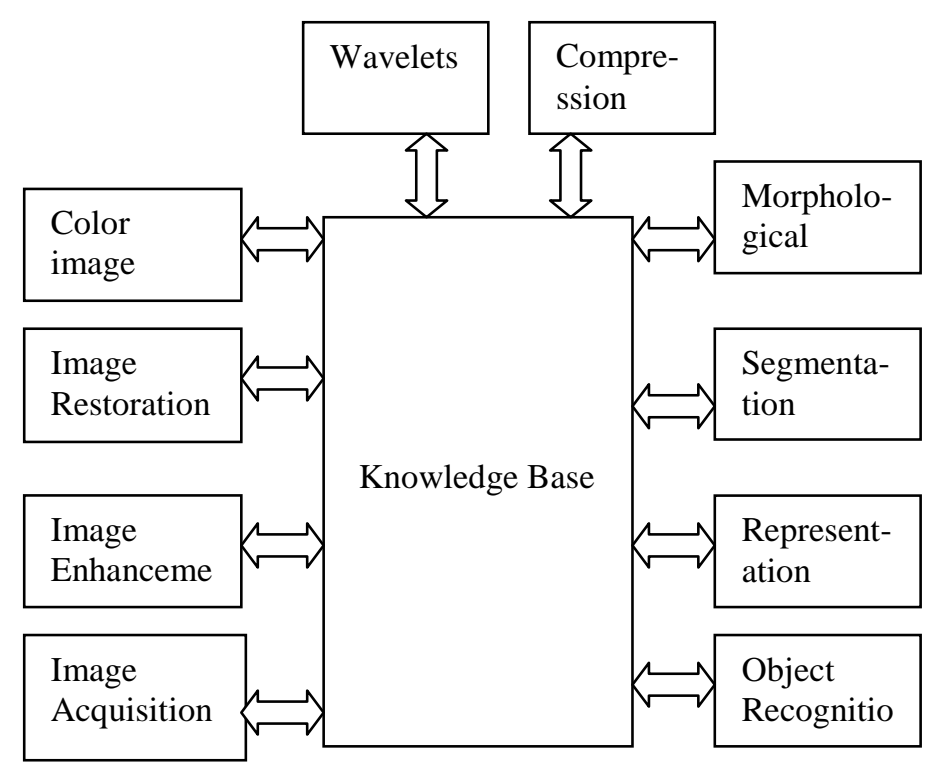

Fig. (b) Steps in digital image processing

The organization is summarized in fig (b). The diagram does not imply that every process is applied to an image. Rather the intention is to convey an idea of all methodologies that can be applied to images for different purpose and possibly with different objective.

Image acquisition is the first process introduces a number of basic digital image concept that are used. The image acquisition stage involves preprocessing such as scaling. Image enhancement is the simplest and most appealing areas of digital image processing. Enhancement simply to highlight certain features of interest in an image. It is a very subjective area of image processing. Image restoration is an area that also deals with improving the appearance of an image. Image restoration is objective, the sense that restoration techniques tend to be based on mathematical or probabilistic model of 


\section{Bhausaheb Shivajirao Shinde, A.R. Dani / IOSR Journal of Engineering (IOSRJEN) \\ www.iosrjen.org}

Vol. 1, Issue 1, pp.066-071

\section{A Model of the Image Restoration Process}

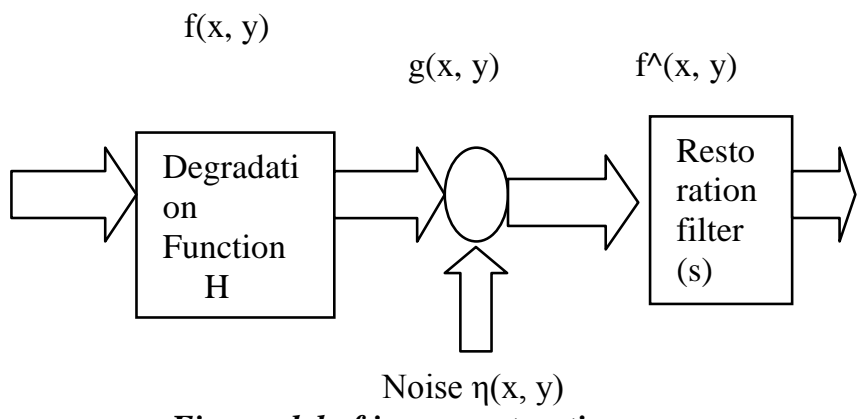

Fig. model of image restoration

A Fig shows, the degradation or restoration process is modeled. A degradation function that, together with an additive noise term, operates on an input image $f(x, y)$ to produce a degraded image $\mathrm{g}(\mathrm{x}, \mathrm{y})$. Given $\mathrm{g}(\mathrm{x}, \mathrm{y})$, some knowledge about the degradation function $\mathrm{H}$, and come knowledge about the additive noise term $\eta(x, y)$, the objective of restoration is to obtain an estimate $\mathrm{f}^{\wedge}(\mathrm{x}, \mathrm{y})$ of the original image. We are estimating as close as possible to the original input image and, in general, the more we know about $\mathrm{H}$ and $\eta$, the closer $\mathrm{f}^{\wedge}(\mathrm{x}, \mathrm{y})$ will be to $\mathrm{f}(\mathrm{x}, \mathrm{y})$. If $\mathrm{H}$ is a linear, positiveinvariant process, then the degraded image is given in the spatial domain by

$$
\mathrm{g}(\mathrm{x}, \mathrm{y})=\mathrm{h}(\mathrm{x}, \mathrm{y}) * \mathrm{f}(\mathrm{x}, \mathrm{y})+\eta(\mathrm{x}, \mathrm{y})
$$

Where $\mathrm{h}(\mathrm{x}, \mathrm{y})$ is the spatial representation of the degradation function and symbol ' $*$ ' indicates spatial convolution. That convolution in the spatial domain is equal to

Multiplication in the frequency domain, so we may write the model in an equivalent frequency domain representation

$$
\mathrm{G}(\mathrm{u}, \mathrm{v})=\mathrm{H}(\mathrm{u}, \mathrm{v}) \mathrm{F}(\mathrm{u}, \mathrm{v})+\mathrm{N}(\mathrm{u}, \mathrm{v})
$$

Where the term in capital letters is the Fourier transforms of the corresponding terms.

We assume that $\mathrm{H}$ is the identity operator and we deal with degradation due to noise.

\section{Why Medical Imaging?}

The internal structure of the human body is not generally visible to the human eyes. However, by various imaging techniques images can be created through which the medical professionals can look into the body to diagnose abnormal conditions and guide the therapeutic procedures. Different medical imaging methods reveal different characteristics of the human body. With each method, image quality and structure visibility can be considerable, depending on characteristics of the imaging equipment, skill of the operator, and compromises with factors such as patient radiation exposure and imaging time.
Noise in Medical Image:It is generally desirable that image brightness is to be uniform except where it changes to form an image. There is a variation in the brightness of a displayed image even when no image detail is present. This variation is usually random and has no particular pattern reducing the image quality specifically when the images are small and have relatively low contrast. This random variation in image brightness is nothing but a noise. All medical images contain some visual noise. The presence of noise gives an image a grainy, textured, or snowy appearance. No imaging method is free of noise, but noise is much more prevalent in certain types of imaging procedures than in others. Most of the imaging methods can create image features that do not represent a body structure or object. These are image artifacts.

In many situations an artifact does not significantly affect object visibility and diagnostic accuracy. But artifacts can obscure a part of an image or may be interpreted as an anatomical feature. A medical image should give an accurate impression of body objects in terms of their size, shape, and relative positions; however, it may introduce distortion of these factors

\section{MEHODOLOGY:}

\section{Noise Models :}

Real images are often degraded by some random errors - This degradation is usually called noise. Noise can occur during image capture transmission or processing and may dependent on or independent of image content. Basically, there are two types of noise models: Noise in the spatial domain (described by the noise probability density function) and noise in the frequency domain described by various Fourier properties of the noise. Now here with we are discussing about the noise is independent of image co-ordinates.

\section{Gaussian Noise:}

Gaussian noise is popular noise approximation. A random variable with Gaussian (normal) distribution has its probability density is given by the Gaussian curve.

The ID case the density function is

$$
\mathrm{P}(\mathrm{x})=1 / \sqrt{ } 2 \prod^{\mathrm{e}-(\mathrm{z}-\mu) 2 / 2 \square 2}
$$

Where $\mu$ is the mean and $\square$ is the standard deviation of random variable. Gaussian noise that occurs in many practical cases.

\section{Additive Noise:}

When an image is transmitted through some communication channel, a noise which is usually independent of the signal occurs. Similar noise arises in video camera. This signal 


\section{Bhausaheb Shivajirao Shinde, A.R. Dani / IOSR Journal of Engineering (IOSRJEN) www.iosrjen.org}

Vol. 1, Issue 1, pp.066-071

independent degradation is called additive noise and can be described by the following models.

$$
F(x, y)=g(x, y)+v(x, y)
$$

Where $\mathrm{v}(\mathrm{x}, \mathrm{y})$ is noise, which independent of input image $\mathrm{g}(\mathrm{x}$, $y)$ and $f(x, y)$ is corrupted image. The noise magnitude depends on the signal magnitude itself.

\section{Multiplicative Noise:}

If the noise magnitude is much higher in comparison with the signal we can write

$$
\mathrm{F}=\mathrm{g}+\mathrm{gv}=\mathrm{g}(1+\mathrm{v})=\mathrm{gv}
$$

The above equation describes the multiplicative noises is the television raster degradation which depends on TV lines in ten area of line this noise is minimal. [Gonzalez, 2005]

\section{Quantization Noise:}

It occurs when insuffient Quantization levels are used for ex 50 levels for monochrome image in this case false contour appear. [Gonzalez, 2005]

\section{Impulsive Noise:}

Impulsive noise means that an image is corrupted with individual noisy pixels whose brightness significantly differs from the neighborhood.

\section{Salt and Pepper Noise:}

It is another type of noise is used to describe saturated impulsive noise an image corrupted with white and/ or a black pixel is an example. Salt and Pepper noise can corrupt binary image.

All the above noises can be added using the IPT function imnoise (). The basic syntax is

$$
\mathrm{G}=\text { imnoise (f, type, parameters) }
$$

Where $\mathrm{f}$ is the input image, type is the type of noise.

Function imnoise () converts the input image to class double in the range $[0,1]$ before adding noise to it. This must be taken account when specifying noise parameter.

- $\mathrm{G}=$ imnoise ( $\mathrm{f}$, Gaussian, m, var) adds Gaussian noise of mean $m$ and variance var to image $f$. Te default is zero mean noise with 0.01 variance.

- $\mathrm{G}=$ imnoise ( $\mathrm{f}$, localvar, $\mathrm{v}$ ) adds zero mean, Gaussian noise of local variance, $V$, to image $f$, where $\mathrm{v}$ is an array of the same size as f containing the desired variance values at each point.

- $\mathrm{G}=$ imnoise (f, 'localvar', image intensity, var) adds zero mean, Gaussian noise to image $f$, where the local variance of the noise, var, is a function of the image intensity values in ' $\mathrm{f}$ '. The image intensity and var arguments are vectors must contain normalized intensity values in the rang $[0,1]$

- $G$ = imnoise (f, 'salt \& pepper', d) corrupts image f with salt $\&$ pepper noise, where $d$ is the noise density (i.e. the present of the image area containing noise values). Thus, approximately $\mathrm{d}^{*}$ numel (f) pixels are affected. The default is 0.05 noise density.
- $\mathrm{G}=$ imnoise (f,' speckle', var) adds multiplicative noise to image $f$ using the equation $g=f+n * f$, where $n$ is uniformly distributed random noise with mean 0 and variance var. The default vale of var $0.04 . \mathrm{G}=$ imnoise (f, 'Poisson') generates Poisson noise from data instead of adding artificial noise to the data. In order to comply with Poisson statistics, the intensities Periodic Noise:

Periodic noise is an image arises typically from electrical and / or electromechanical inferences during the image acquisition. This is the only type of spatially dependent noise. Periodic noise typically handled in an image by filtering in the frequency domain.

The model of periodic noise is a 2-D sinusoid with equation

$\mathrm{R}(\mathrm{x}, \mathrm{y})=\mathrm{A} \sin \left[2 \prod \mathrm{u} 0(\mathrm{x}+\mathrm{Bx}) / 2 \prod \mathrm{v} 0(\mathrm{y}+\mathrm{by}) / \mathrm{N}\right]$

Where $\mathrm{A}$ is the amplitude, $\mathrm{u} 0$ and $\mathrm{v} 0$ determines the sinusoidal frequencies with respect to the $\mathrm{x}$ and $\mathrm{y}$ axies respectively and $\mathrm{Bx}$ and By are phase's displacements with respect to the origin.

\section{DISCUSSION:}

Different medical images like MRI, Cancer, $\mathrm{x}$-ray and brain images have been studied. After finding the Gaussian noise in MRI image the various filtering techniques like Median filter, Adaptive filter and Average filter have been applied.

It is found that the Adaptive filter works better for the gaussian noise .Similarly after finding the Gaussian noise in cancer image the various filtering techniques have been applied and it is found that the adaptive filter works better for the noisy image. After finding the Gaussian noise in X-ray image various filtering techniques have been applied and it is found that the adaptive filter works better for the X-ray noisy image. After finding the Gaussian noise in brain image various filtering techniques have been applied and it is found that the adaptive filter works better for the noisy image. Similarly after finding the salt and pepper noise in MRI image various filtering techniques have been applied and it is found that the adaptive filter works better for the noisy image. After finding the salt and pepper noise in Cancer image various filtering techniques have been applied and it is found that the median filter works better for the noisy image. After finding the salt and pepper noise in X-ray image various filtering techniques have been applied and it is found that the median filter works better for the noisy image. After finding the salt and pepper noise in Brain image various filtering techniques have been applied and it is found that the adaptive filter works better for the noisy image.

So we can say that there are no filtering techniques which will work better for all types of noises like gaussian noise, salt and pepper nosie, speckle nosie and Poisson noise.

\section{CONCLUSION:}

In this work we have taken different medical images like MRI, Cancer, X-ray and Brain for detecting noises. We have 
Vol. 1, Issue 1, pp.066-071

detected various noises like Gaussian, Salt \& Pepper, Speckle, and Poisson and also removed these noises from the above medical images by applying the various filtering techniques like Median Filtering, Adaptive Filtering and Average Filtering. Through this work we have observed that the choice of filters for de-noising the medical images depends on the type of noise and type of filtering technique, which are used. It is remarkable that this saves the processing time. This experimental analysis will improve the accuracy of MRI, Cancer, X-ray and Brain images for easy diagnosis. The results, which we have achieved, are more useful and they prove to be helpful for general medical practitioners to analyze the symptoms of the patients with ease.

\section{REFERENCES:}

[1] Rafael C.Gonzalez \& Richard E.Woods, "Digital Image Processing", Second edition,

[2] Rafael C. Gonzalez \& Richard E.Woods, "Digital Image Processing using MATLAB", Pearson education

[3] Milan Sonka et.al,"'Image Processing Analysis and Machine Vision", International Thomson computer press,

[4] Kenneth R.Castleman, "Digital Image processing", PHI Adrian Low ," Computer Vision \& Image Processing", McGraw Hill

[5] Stewan W.Smit,"Scientist and Engineers Guide to Digital Image processing", Second Edition,

[6] K. V. Kale et.al "Noise Removal of Medical Images through Decimated and Un-Decimated Wavelet Transform", IEEE 1st International Conference On signal And Image processing, Hubli, India,

[7] S.S.Gornale et.al," Evaluation \& selection of wavelet filters for de-noising medical Images using Stationary wavelet Transform (SWT)"

[8] Shalkoff R. J, , John wiley and sons, New York, "Digital Image Processing and computer vision".

Shinde Bhausaheb: I have completed my M.C.S.(Master Of Computer Science) from Pune University, M.Phil. Also Register to Ph.D in Sighania University, Rajastan. Currently I am working in R.B.N.B. College as Head of Computer Science Department having 12 years of expert as well as Lecturer experience. 